\title{
„An der Uni habe ich mehr Freiheiten als in der Praxis"
}

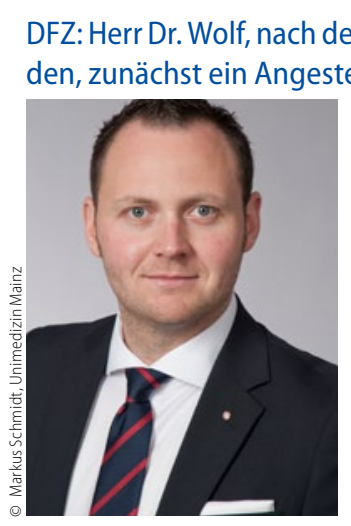

Dr. Thomas Wolf
Wolf: Ich konnte mich schon währen des Studiums für eine Karriere in der Wissenschaft begeistern, weil ich es faszinierend fand, mit Forschung in Berührung zu kommen. ,Mit dem Wissen wächst der Zweifel', hat ja schon Goethe gesagt. Ich finde es spannend, und es macht mir Spaß, neues Wissen zu kreieren: Ob es darum geht, zum Wohle des Patienten neue Techniken, Innovationen oder Geräte mitzuentwickeln und zu verbessern, oder bessere Heilmethoden zu erforschen. Und so habe ich nach meinem Examen im Jahr 2011 direkt an der Uni Mainz als wissenschaftlicher Mitarbeiter angefangen, um in Forschung und Lehre tätig zu sein. Gerade befinde ich mich im Habilitationsverfahren.

DFZ: Welche Vorteile sehen Sie insbesondere in Ihrer Tätigkeit für die Universitätsmedizin Mainz im Gegensatz zu einer Tätigkeit als Angestellter in einer Praxisgemeinschaft?

Wolf: An der Uni habe ich mehr Freiheiten als in der Praxis, wo der Fokus ja hauptsächlich auf der Patientenbehandlung liegt. Hier kann ich eine Vielzahl von Funktionen ausüben, etwa auch als Strahlenschutz- oder Unterrichtsbeauftragter. Als Letzterer bin ich für die Vorbereitung und Durchführung von Kursen und Vorlesungen mit verantwortlich. Ich kann mir zudem das Betätigungsfeld aussuchen, auf das ich den Fokus setze - und optimal ist es, wenn man dann noch wie ich das Glück hat, einen guten Mentor zu haben.

In der Praxis kann man ja leider nur sehr schwierig Forschung betreiben, das ist kein Vergleich zur Uniklinik. Aber ich mache auch Praxisvertretungen, um den Vergleich zu sehen und einen Fuß in der Berufspraxis zu behalten. Zudem werden in den Unikliniken die zehn Prozent der am schwierigsten zu behandelnden Patienten versorgt: Multimorbide Patienten und komplexe Fälle müssen Hochschulambulanzen mit abdecken, weil sie andere Möglichkeiten haben als Praxen.

DFZ: Könnten Sie sich vorstellen, sich eines Tages doch selbstständig zu machen? Unter welchen Umständen wäre dies vorstellbar? Wolf: Momentan kann ich mir nicht vorstellen, der Uni komplett den Rücken zu kehren. Mein Ziel nach der Habilitation ist, eine Oberarztstelle anzunehmen und möglicherweise eines Tages einen Lehrstuhl zu leiten. Eine Alternative, die einige Kollegen in Betracht gezogen haben, wäre eine Kombination aus Uni und Praxis. Aber zum jetzigen Zeitpunkt ist das für mich weder absehbar, noch steht dies zur Debatte.

DFZ: Inwiefern ist der FVDZ auch für Angestellte wichtig?
Hier steht eine Anzeige. Springer
Wolf: Der FVDZ bietet auch angestellten Zahnärzten eine standespolitische Plattform. Der FVDZ ist dabei ein bedeutsamer Wegweiser und hilft den Angestellten auf dem Weg zur Selbstständigkeit. Dabei setzt sich der FVDZ gesundheitspolitisch für bessere Rahmenbedingungen ein. Zudem bietet der Freie Verband Deutscher Zahnärzte etliche Service-Leistungen, wie zum Beispiel einen Praxispool, wo sich Angestellte beziehugsweise Niederlassungswillige mit Praxisabgebern zusammenfinden, eine Akademie mit Fortbildungen zu Themen wie Recht, Betriebswirtschaft, Praxismanagement und Fachthemen sowie verschiedene Kongresse auf Sylt, in Usedom (im Sommer) und Ischgl (im Winter). Auch im Praxishandbuch können bereits Angestellte die notwendigen Informationen zur Praxis finden. Es gibt zudem tolle Programme schon für Studierende, für „young dentists“ und auch für Wissensbegeisterte im Wissenschaftlichen Info-Dienst mit verständlich aufbereiteten aktuellen Fachthemen.

Interview: Maike Edda Raack 